Document downloaded from:

http://hdl.handle.net/10251/150310

This paper must be cited as:

Pellegrino, D.; Rueda, P.; Sánchez Pérez, EA. (2016). Improving integrability via absolute summability: a general version of Diestel s Theorem. Positivity. 20(2):369-383. https://doi.org/10.1007/s11117-015-0361-5

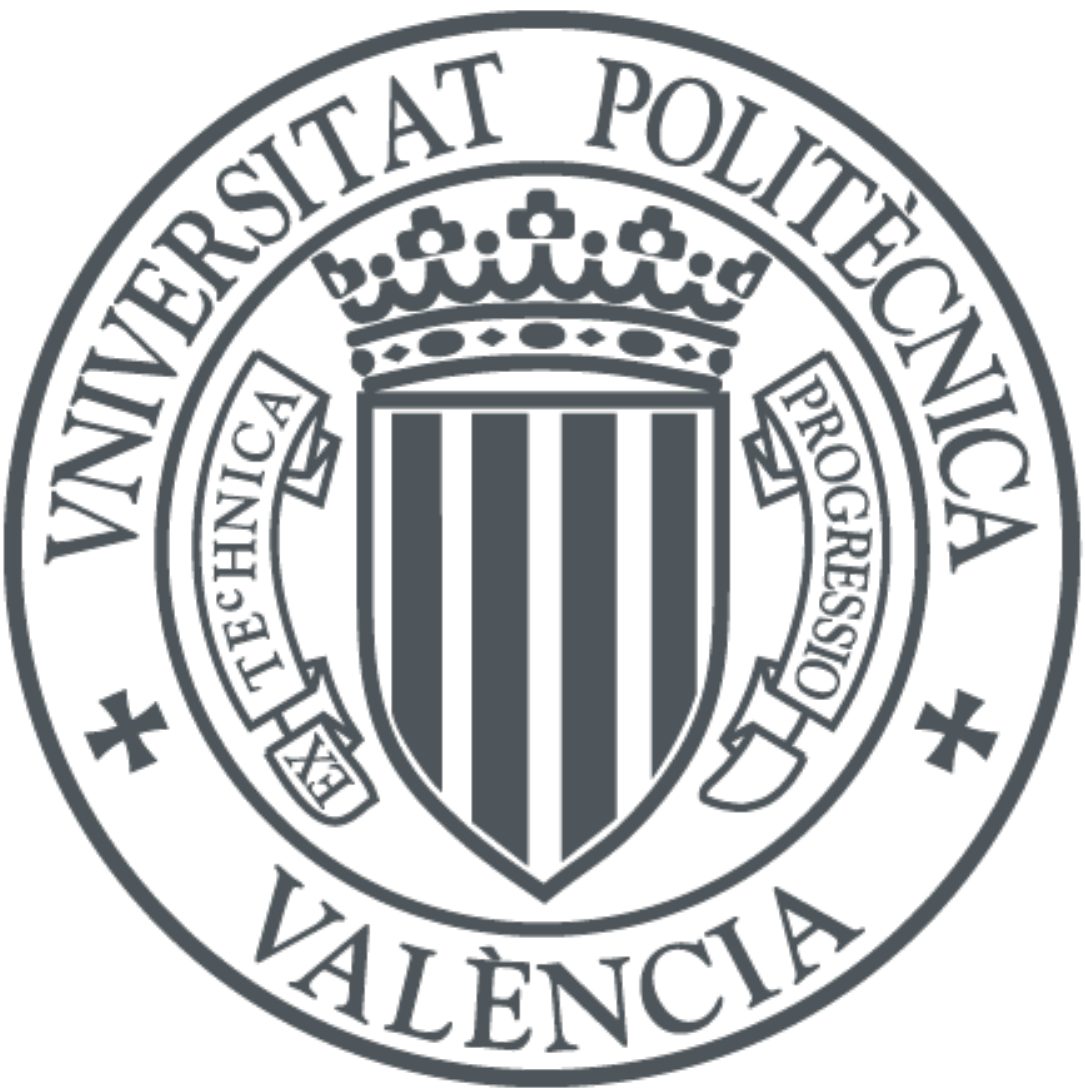

The final publication is available at

https://doi.org/10.1007/s11117-015-0361-5

Copyright Springer-Verlag

Additional Information 


\title{
Improving integrability via absolute summability: a general version of Diestel's Theorem
}

\author{
D. Pellegrino \\ Departamento de Matemática \\ Universidade Federal da Paraíba \\ 58.051-900, João Pessoa, Brazil \\ e-mail: dmpellegrino@gmail.com \\ P. Rueda ${ }^{1}$ \\ Departamento de Análisis Matemático \\ Universidad de Valencia \\ 46100 Burjassot - Valencia, Spain \\ e-mail: pilar.rueda@uv.es, phone: 0034963543907 fax: 0034963544723 \\ E.A. Sánchez-Pérez \\ Instituto Universitario de Matemática Pura y Aplicada \\ Universitat Politècnica de València \\ Camino de Vera s/n, 46022 Valencia, Spain \\ e-mail: easancpe@mat.upv.es
}

AMS subject classification(2010): Primary 46E40, Secondary 47B10

Keywords: absolutely summing operator, absolutely continuous operator, Pettis integrable function, Bochner integrable function. ${ }^{1}$

\begin{abstract}
A classical result by J. Diestel establishes that the composition of a summing operator with a (strongly measurable) Pettis integrable function gives a Bochner integrable function. In this paper we show that a much more general result is possible regarding the improvement of the integrability of vector valued functions by the summability of the operator. After proving a general result, we center our attention in the particular case given by the $(p, \sigma)$-absolutely continuous operators, that allows to prove a lot of special results on integration improvement for selected cases of classical Banach spaces - including $C(K), L^{p}$ and Hilbert spaces - and operators $-p$-summing, $(q, p)$-summing and $p$-approximable operators-.
\end{abstract}

\footnotetext{
${ }^{1}$ Corresponding author
} 


\section{Introduction}

Let $X$ and $Y$ be Banach spaces. Let $(\Omega, \Sigma, \mu)$ be a finite, positive, non-atomic measure space, and let $1 \leq p<\infty$. Consider the space $\mathcal{P}_{p}(\mu, X)$ of all (equivalence classes of) strongly measurable Pettis $p$-integrable functions with respect to $\mu, f: \Omega \rightarrow X$ with the norm

$$
\|f\|_{\mathcal{P}_{p}}:=\sup \left\{\left(\int\left|x^{\prime}(f(w))\right|^{p} d \mu(w)\right)^{1 / p}: x^{\prime} \in X^{*},\left\|x^{\prime}\right\| \leq 1\right\},
$$

and let $\mathcal{B}_{p}(\mu, X)$ be the space of all Bochner $p$-integrable functions with respect to $\mu, f: \Omega \rightarrow X$ with the norm

$$
\|f\|_{\mathcal{B}_{p}}:=\left(\int\|f(w)\|^{p} d \mu(w)\right)^{1 / p} .
$$

A linear operator $T: X \longrightarrow Y$ is absolutely $p$-summing if $\left(T\left(x_{n}\right)\right)_{n=1}^{\infty}$ is absolutely $p$-summable in $Y$ whenever $\left(x_{n}\right)_{n=1}^{\infty}$ is weakly $p$-summable in $X$. Diestel proved in 1972 that an absolutely 1-summing (from now on simply called absolutely summing) operator not only improves the summability of sequences but also the integrability of functions in the following sense (see [3]): Consider $u \in \mathcal{L}(X, Y)$ and let $\tilde{u}: \mathcal{P}_{1}(\mu, X) \rightarrow \mathcal{P}_{1}(\mu, Y)$ be given by $(\tilde{u} f)(w):=u(f(w))$, $w \in \Omega$ and $f \in \mathcal{P}_{1}(\mu, X)$. Then $\tilde{u}$ belongs to $\mathcal{L}\left(\mathcal{P}_{1}(\mu, X) ; \mathcal{B}_{1}(\mu, Y)\right)$ if and only if $u$ is absolutely summing.

The transcendence of absolutely summing operators and their connection, provided by Diestel, with integrability has produced the appearance of several works dealing with improvements of integrability of functions via composition with absolutely summing operators. For instance, Rodríguez [15] has proved that if $u$ is absolutely summing then $\tilde{u}$ maps Dunford integrable functions to scalarly equivalent functions to Bochner integrable ones. Furthermore, he has proved that $U$ maps Birkhoff integrable or McShane integrable functions to Bochner integrable functions (see [15] for definitions and for some other related works). Our aim is to show that a variant of this improvement is fulfilled by $R S$ abstract summing operators in the sense of [1] (see also [11]). This general result has many consequences. We will center our attention in a particular case, that contains the classical result by Diestel and let to consider it as a starting point of a scale of results concerning the relation between $(p, q)$-summability of $u$ and the improvement of integrability provided by $\tilde{u}$. In order to do it, our main tool is the study of the improvement of integrability given by operators belonging to a particular class of interpolated operator ideals of summing operators: the so called ideals of $(p, \sigma)$-absolutely continuous operators (see [7, 9, 10]). We will need to introduce also a new definition of integrability of strongly measurable (vector valued) functions that is given by some interpolation formula between the Bochner integral and the Pettis integral.

However, we establish first our main result - Theorem 3 - in more abstract terms. The reason is that several recent papers have shown that, in fact, the main arguments that prove the important results on ideals of operators improving summability, can be formulated and proved in a very general setting, and all 
the properties that hold for the classical case work also in this abstract framework. This also happens in the case of the problem analyzed in the present paper. In [1], the definition of $R S$-abstract p-summing operator is given, and a generalized Pietsch's Domination Theorem is proved for it. In fact, it is proved that all known Pietsch type theorems are particular cases of this one, including the multilinear cases. For this reason, this is the definition that we adopt. In [11], an even more general definition of $R S$-abstract summing operator is given removing two hypothesis and still preserving the Domination Theorem. In this direction, a general framework regarding all these arguments is presented in [12], and it is shown how can a lot of results of this kind be considered as particular instances of a general principle; the interested reader can find there - and in the references in this paper-, a general explanation of the general topics and techniques in operator theory that are covered with the $R S$-abstract summing operators.

In the second part of the paper (Section 5), we present a lot of examples and applications of our results for particular sets of classical Banach spaces and operators that are better known than the $(p, \sigma)$-absolutely continuous operators. We will follow mainly the results in [10], and also the ones in $[7,8,9]$.

\section{Basic concepts and notation}

We will use standard Banach space notation. If $1 \leq p \leq \infty$, we will write $p^{\prime}$ for the extended real number that satisfies that $1 / p+1 / p^{\prime}=1$. Unless stated otherwise $X, Y, E$ and $F$ will be Banach spaces. $\mathcal{L}(X, Y)$ denotes as usual the space of linear and continuous operators from $X$ to $Y$. We will write $X^{*}$ for the dual of $X$ and $B_{X^{*}}$ denotes the closed unit ball of $X^{*}$ endowed with the weak star topology. Our fundamental references for the general theory of summing operators and general operator ideals are $[2,4,13]$. The definition of $R S$-abstract summing operator is the following.

Consider arbitrary sets $X$ and $Y$ and $E$, let $\mathcal{H}$ be a family of mappings from $X$ to $Y, G$ be a Banach space and $K$ be a compact Hausdorff topological space.

Let $R: K \times E \times G \rightarrow[0, \infty)$ and $S: \mathcal{H} \times E \times G \rightarrow[0, \infty)$ be mappings such that the mapping $R_{x, b}: K \rightarrow[0, \infty), R_{x, b}(\varphi)=R(\varphi, x, b)$ is continuous for each $x \in E$ and $b \in G$.

Using these elements, the following notion is introduced in [1, Def.2.1] and [11].

Definition 1 Let $R$ and $S$ as above and $0<p<\infty$. A mapping $f \in \mathcal{H}$ is said to be $R S$-abstract p-summing if there is a constant $C_{1}>0$ such that

$$
\left(\sum_{i=1}^{n} S\left(f, x_{i}, b_{i}\right)^{p}\right)^{\frac{1}{p}} \leq C_{1} \sup _{\varphi \in K}\left(\sum_{i=1}^{n} R\left(\varphi, x_{i}, b_{i}\right)^{p}\right)^{\frac{1}{p}},
$$

for all $x_{1}, \ldots, x_{n} \in E, b_{1}, \ldots, b_{n} \in G$ and $n \in \mathbb{N}$. 
A particular instance of this abstract notion of summability is given by the interpolated class of linear operators cited above. Let $1 \leq p<\infty$ and $0 \leq \sigma<1$. A linear operator $T: X \rightarrow Y$ is said to be $(p, \sigma)$-absolutely continuous if for every $x_{1}, \ldots, x_{n} \in X$,

$$
\left(\sum_{i=1}^{n}\left\|T\left(x_{i}\right)\right\|^{\frac{p}{1-\sigma}}\right)^{\frac{1-\sigma}{p}} \leq C \sup _{x^{\prime} \in B_{X^{*}}}\left(\sum_{i=1}^{n}\left(\left|\left\langle x_{i}, x^{\prime}\right\rangle\right|^{1-\sigma}\left\|x_{i}\right\|^{\sigma}\right)^{\frac{p}{1-\sigma}}\right)^{\frac{1-\sigma}{p}} .
$$

The space of all linear operators from $X$ to $Y$ satisfying this property will be denoted in this paper by $\Pi_{p}^{\sigma}(X, Y)$. It is a normed space, being $\pi_{p}^{\sigma}(T)$ the norm computed as the infimum of all the constants $C>0$ in the inequality above (see $[7,9,10])$. It is easy to see that

$$
\Pi_{p_{1}}^{\sigma_{1}} \subseteq \Pi_{p_{2}}^{\sigma_{2}}
$$

for $1 \leq p_{1} \leq p_{2}<\infty$ and $0 \leq \sigma_{1} \leq \sigma_{2}<1$ (see [10, Prop. 3.3]; see also [7]).

The usual space of $(p, q)$-summing operators will be relevant in this paper too, for $1 \leq q \leq p<\infty$. This is given by all linear operators $T: X \rightarrow Y$ for which there exists a constant $C>0$ depending on $T$ such that for every $x_{1}, \ldots, x_{n} \in X$

$$
\left(\sum_{i=1}^{n}\left\|T\left(x_{i}\right)\right\|^{p}\right)^{\frac{1}{p}} \leq C \sup _{x^{\prime} \in B_{X^{*}}}\left(\sum_{i=1}^{n}\left|\left\langle x_{i}, x^{\prime}\right\rangle\right|^{q}\right)^{\frac{1}{q}}
$$

We will use the symbol $\Pi_{p, q}(X, Y)$ to denote the component of this operator ideal when the linear operators from $X$ to $Y$ are considered, and the norm for such an operator $T$ will be denoted by $\pi_{p, q}(T)$ and defined as the infimum of all constants $C$ as above. For $p=q$, we get the operator ideal of $p$-summing operators; the notation will be in this case $\Pi_{p}(X, Y)$ and $\pi_{p}(T)$.

The following well-known result will be used several times in this paper. Its proof can be found in [10, Prop.4.2].

Lemma 2 Let $1 \leq p<\infty$ and $0 \leq \sigma<1$. Then $\Pi_{p} \subseteq \Pi_{\frac{p}{1-\sigma}} \subseteq \Pi_{p}^{\sigma} \subseteq \Pi_{\frac{p}{1-\sigma}, p}$.

\section{A characterization of $R S$-abstract $p$-summing operators in terms of integrability.}

Let $K$ be a compact set and $H$ a space of operators. Let $E$ and $G$ be Banach spaces. Let $S: H \times E \times G \rightarrow \mathbb{R}^{+}$and $R: K \times E \times G \rightarrow \mathbb{R}^{+}$be functions such that

$$
S(u, 0, b)=S(v, x, 0)=0=R(\phi, 0, b)=R(\varphi, x, 0)
$$

for all $u, v \in H, x \in E, b \in G, \phi, \varphi \in K$. Let $(\Omega, \Sigma, \mu)$ be a non-atomic finite measure space, and let $M(\Omega, E)$ and $N(\Omega, G), i=1,2$, be sets of strongly measurable (classes of $\mu$-a.e. equal) functions with values in $E$ and $G$ respectively. Consider subsets $M \subset M(\Omega, E)$ and $N \subset N(\Omega, G)$ that contain the 
simple functions. We define the real functions $\tilde{S}: H \times M \times N \times \Omega \rightarrow \mathbb{R}^{+}$and $\tilde{R}: K \times M \times N \times \Omega \rightarrow \mathbb{R}^{+}$given by

$$
\tilde{S}(u, f, g, w):=S(u, f(w), g(w))
$$

and

$$
\tilde{R}(\varphi, f, g, w):=R(\varphi, f(w), g(w)) .
$$

As our objective is to show how $R S$-abstract summing operators improve the integrability of functions, we will assume that the function $\tilde{R}_{\varphi, f, g}(w):=$ $\tilde{R}(\varphi, f, g, w)$ is $\mu$-integrable for all $\varphi \in K, f \in M$ and $g \in N$. Then, under this assumption, our aim is to relate summability of $u$ with integrability of $\tilde{S}_{f, g}(w):=\tilde{S}(u, f, g, w), f \in M$ and $g \in N$.

Notice that the definition of $u$ being $R S$-abstract $p$-summing is equivalent to the fact that there is $C>0$ such that for finite families $x_{1}, \ldots, x_{n} \in E$, $b_{1}, \ldots, b_{n} \in G$ and $a_{1,1}, \ldots, a_{1, n}, \ldots, a_{n, 1}, \ldots, a_{n, n} \in \mathbb{R}^{+}$,

$$
\left(\sum_{i, j=1}^{n} a_{i, j} S\left(u, x_{i}, b_{j}\right)^{p}\right)^{1 / p} \leq C \sup _{\varphi \in K}\left(\sum_{i, j=1}^{n} a_{i, j} R\left(\varphi, x_{i}, b_{j}\right)^{p}\right)^{1 / p} .
$$

In order to see this, a tricky approximation argument using rational numbers is needed. Take $x_{1}, \ldots, x_{n} \in E$ and $b_{1}, \ldots, b_{n} \in G$ as above and fix positive integers $\alpha_{1,1}, \ldots, \alpha_{1, n}, \ldots, \alpha_{n, 1}, \ldots, \alpha_{n, n}$. Consider the original inequality in the definition of $R S$-abstract $p$-summing operator repeating $\alpha_{i, j}$ times each term with subindexes $i$ and $j$ in both sums. This gives the desired inequality for positive integers. After choosing the adequate integers and dividing by the right denominator the expressions in both sides of the inequality, we get it for rational numbers. Finally, using the density of the set of rationals, we obtain the relation for arbitrary positive real numbers $a_{i, j}$. The main idea of this argument appeared in [5], and it has been used for example in [1, 12].

Theorem 3 An operator $u: X \rightarrow Y$ is RS-abstract summing if and only if there is a constant $C>0$ such that

$$
\int_{\Omega} \tilde{S}(u, f, g, w) d \mu(w) \leq C \sup _{\varphi \in K} \int_{\Omega} \tilde{R}(\varphi, f, g, w) d \mu(w)
$$

for all simple functions $f \in M$ and all simple functions $g \in N$.

Proof. $(\Rightarrow)$ Take a pair of simple functions $f=\sum_{i=1}^{n} x_{i} \chi_{A_{i}} \in M$ and $g=$ $\sum_{j=1}^{m} b_{j} \chi_{B_{j}} \in N$, with $x_{1}, \ldots, x_{n} \in E, b_{1}, \ldots, b_{m} \in G, A_{1}, \ldots, A_{n}, B_{1}, \ldots, B_{m} \in$ $\Sigma$. Then, since we are assuming both $u$ is $R S$-abstract summing and condi- 
tion (2),

$$
\begin{aligned}
\int_{\Omega} \tilde{S}(u, f, g, w) d \mu(w) & =\int_{\Omega} S(u, f(w), g(w)) d \mu(w) \\
& =\sum_{i=1}^{n} \sum_{j=1}^{m} S\left(u, x_{i}, b_{j}\right) \mu\left(A_{i} \cap B_{j}\right) \\
& \leq C \sup _{\varphi \in K} \sum_{i=1}^{n} \sum_{j=1}^{m} R\left(\varphi, x_{i}, b_{j}\right) \mu\left(A_{i} \cap B_{j}\right) \\
& =C \sup _{\varphi \in K} \int_{\Omega} R\left(\varphi, \sum_{i=1}^{n} x_{i} \chi_{A_{i}}, \sum_{j=1}^{m} b_{j} \chi_{B_{j}}\right) d \mu(w) \\
& =C \sup _{\varphi \in K} \int_{\Omega} \tilde{R}(\varphi, f, g, w) d \mu(w) .
\end{aligned}
$$

$(\Leftarrow)$ Consider $x_{1}, \ldots, x_{n} \in E$ and $b_{1}, \ldots, b_{n} \in G$. Since the measure is nonatomic, there are pairwise disjoint measurable sets $A_{1}, \ldots, A_{n}$ in $\Sigma$ such that $\mu\left(A_{1}\right)=\ldots=\mu\left(A_{n}\right)=: \alpha>0$. Then

$$
\begin{aligned}
\alpha \sum_{i=1}^{n} S\left(u, x_{i}, b_{i}\right) & =\sum_{i=1}^{n} \mu\left(A_{i}\right) S\left(u, x_{i}, b_{i}\right)=\int_{\Omega} \sum_{i=1}^{n} S\left(u, x_{i}, b_{i}\right) \chi_{A_{i}}(w) d \mu(w) \\
& =\int_{\Omega} S\left(u, \sum_{i=1}^{n} x_{i} \chi_{A_{i}}(w), \sum_{i=1}^{n} b_{i} \chi_{A_{i}}(w)\right) d \mu(w) \\
& =\int_{\Omega} \tilde{S}\left(u, \sum_{i=1}^{n} x_{i} \chi_{A_{i}}, \sum_{i=1}^{n} b_{i} \chi_{A_{i}}, w\right) d \mu(w) \\
& \leq C \sup _{\varphi \in K} \int_{\Omega} \tilde{R}\left(u, \sum_{i=1}^{n} x_{i} \chi_{A_{i}}, \sum_{i=1}^{n} b_{i} \chi_{A_{i}}, w\right) d \mu(w) \\
& =C \sup _{\varphi \in K} \int_{\Omega} \sum_{i=1}^{n} R\left(\varphi, x_{i}, b_{i}\right) \chi_{A_{i}}(w) d \mu(w) \\
& =C \sup _{\varphi \in K} \sum_{i=1}^{n} R\left(\varphi, x_{i}, b_{i}\right) \mu\left(A_{i}\right)=\alpha C \sup _{\varphi \in K} \sum_{i=1}^{n} R\left(\varphi, x_{i}, b_{i}\right) .
\end{aligned}
$$

The proof is done.

One of the main examples of Theorem 3 is Diestel's result [3], where $u$ : $X \rightarrow Y$ is absolutely summing if and only if its associated composition operator carries strongly measurable Pettis integrable functions to Bochner integrable functions. It can be easily seen that it is a particular case of our theorem, once we consider the completion of simple functions to the space of Pettis integrable functions. 
Corollary 4 Let $X$ and $Y$ be Banach spaces, and let $u: X \rightarrow Y$ be a continuous linear operator. Then $u$ is absolutely summing if and only if $\tilde{u}: \mathcal{P}_{1}(\mu, X) \rightarrow$ $\mathcal{B}_{1}(\mu, Y)$ is well-defined and continuous.

Proof. Consider $E=X, K=B_{X^{*}}$ and $G=\mathbb{K}$ (the scalar field). Take $\mathcal{H}=\mathcal{L}(X, Y)$ the space of all continuous linear operators from $X$ into $Y$ and define $R$ and $S$ by:

$$
\begin{gathered}
R: B_{X^{*}} \times X \times \mathbb{K} \longrightarrow[0, \infty), R(\varphi, x, \lambda)=|\lambda||\varphi(x)| \\
S: \mathcal{L}(X, Y) \times X \times \mathbb{K} \longrightarrow[0, \infty), S(T, x, \lambda)=|\lambda|\|T(x)\| .
\end{gathered}
$$

With $R$ and $S$ so defined, a linear operator $u: X \longrightarrow Y$ is $R S$-abstract 1summing if and only if it is absolutely summing. The fact that $\tilde{u}: \mathcal{P}_{1}(\mu, X) \rightarrow$ $\mathcal{P}_{1}(\mu, Y)$ is continuous and the density of the simple functions in $\mathcal{P}_{1}(\mu, X)$ gives the result. The reader can find this argument written in a more precise way in the proof of the forthcoming Theorem 5 .

\section{The case of $(1, \sigma)$-absolutely continuous oper- ators}

This section is devoted to $(1, \sigma)$-absolutely continuous operators. Our aim is to show the power of our main result - Theorem 3 - by showing that the classical result by Diestel (Corollary 4) is in fact an extreme case of a chain of results that characterize integrability of the functions in terms of the summability properties of the operator $u$. The idea is that absolutely summing operators are the starting point (for $\sigma=0$ ) of a series — ordered by inclusion - of classes of linear operators, whose ending point (for $\sigma=1$ ) is the class of all continuous linear operators. The class that holds the position $\sigma$, for $0 \leq \sigma \leq 1$, of this chain is the class of $(1, \sigma)$-absolutely continuous operators. According to this interpolating idea, we introduce the space $\mathcal{P}_{p}^{\sigma}(\mu, X)$ of Pettis $(p, \sigma)$-integrable functions, that can be considered as the interpolated class, for $0 \leq \sigma \leq 1$ and $1 \leq p<\infty$, between $\mathcal{P}_{p}(\mu, X)$ and $\mathcal{B}_{p}(\mu, X)$.

Our aim is to show that an operator $u: X \rightarrow Y$ is $(1, \sigma)$-absolutely continuous if and only if its associated composition operator $\tilde{u}: \mathcal{P}_{1}(\mu, X) \rightarrow \mathcal{P}_{1}(\mu, Y)$ carries Pettis $(1 /(1-\sigma), \sigma)$-integrable functions to $1 /(1-\sigma)$-Bochner integrable functions. Several new results concerning the improvement of integrability via summability of operators are derived.

We need first some definitions. Let $(\Omega, \Sigma, \mu)$ be a non-atomic finite measure space. Consider $0 \leq \sigma \leq 1$ and $1 \leq p<\infty$, and the space $\mathcal{S}_{p}^{\sigma}(\mu, X)$ of all equivalence classes with respect to $\mu$ of simple functions with values in the Banach space $X$. Since all of the functions in it are simple, they satisfy that

$$
\left(\left|\left\langle f(w), x^{\prime}\right\rangle\right|^{1-\sigma}\|f(w)\|^{\sigma}\right)^{p} \in L^{1}(\mu)
$$

for all $x^{\prime} \in X^{*}$, and

$$
\Phi_{p, \sigma}(f):=\sup _{x^{\prime} \in B_{X^{*}}}\left(\int\left(\left|\left\langle f(w), x^{\prime}\right\rangle\right|^{1-\sigma}\|f(w)\|^{\sigma}\right)^{p} d \mu\right)^{1 / p}<\infty .
$$


A seminorm for this space can be given by the convexification $\|\cdot\|_{p, \sigma}$ of $\Phi_{p, \sigma}$ defined by

$$
\|f\|_{p, \sigma}:=\inf \left\{\sum_{i=1}^{n} \Phi_{p, \sigma}\left(f_{i}\right): f=\sum_{i=1}^{n} f_{i}\right\},
$$

for $f \in \mathcal{S}_{p}^{\sigma}(\mu, X)$. Since for all $p$ and $\sigma,\|\cdot\|_{\mathcal{P}_{p}} \leq \Phi_{p, \sigma}(\cdot)$, we have that $\|\cdot\|_{p, \sigma}$ is in fact a norm, and $\mathcal{S}_{p}^{\sigma}(\mu, X) \subseteq \mathcal{P}_{p}(\mu, X)$ continuously for all $0 \leq \sigma \leq 1$.

Write $\overline{S_{p}^{\sigma}(\mu, X)}$ for the completion of $\left(S_{p}^{\sigma}(\mu, X),\|\cdot\|_{p, \sigma}\right)$. Define the space $\mathcal{P}_{p}^{\sigma}(\mu, X)$ as the subspace of the elements of $\overline{S_{p}^{\sigma}(\mu, X)}$ that can be represented by a function in $\mathcal{P}_{p}(\mu, X)$. That is, $\mathcal{P}_{p}^{\sigma}(\mu, X)$ is defined by the elements $z \in$ $\overline{S_{p}^{\sigma}(\mu, X)}$ satisfying that

(1) there is a Cauchy sequence $\left(f_{n}\right)$ in $S_{p}^{\sigma}(\mu, X)$ converging to $z$, and also

(2) $\left(f_{n}\right)$ converges to a strongly measurable Pettis $p$-integrable (class of) function(s) $f$ in $\mathcal{P}_{p}(\mu, X)$.

A direct argument using the continuous inclusion of $S_{p}^{\sigma}(\mu, X)$ in $\mathcal{P}_{p}(\mu, X)$ shows that there is a unique function $f$ as above for each $z \in \mathcal{P}_{p}^{\sigma}(\mu, X)$, and so the definition of $f$ does not depend on the Cauchy sequence $\left(f_{n}\right)$. Therefore, this space can be considered as a function space endowed with the norm induced by $\overline{S_{p}^{\sigma}(\mu, X)}$.

Given $0 \leq \sigma<1$, the case $p=1 /(1-\sigma)$ will be relevant for the applications. The case $p=1$ and $\sigma=0$ gives the classical space of strongly measurable Pettis integrable functions. We will use the fact that given a continuous linear operator $u: X \rightarrow Y$, then $\tilde{u}: \mathcal{P}_{1}(\mu, X) \rightarrow \mathcal{P}_{1}(\mu, Y)$ given by $\tilde{u}(f)=u \circ f$, is well-defined and continuous (see the proof of the theorem in [3]).

Theorem 5 Let $0 \leq \sigma \leq 1$. An operator $u: X \rightarrow Y$ is $(1, \sigma)$-absolutely continuous if and only if the composition operator $\tilde{u}: \mathcal{P}_{1 /(1-\sigma)}^{\sigma}(\mu, X) \rightarrow \mathcal{B}_{1 /(1-\sigma)}(\mu, Y)$ given by $\tilde{u}(f):=u \circ f$ is well defined and continuous. In this case,

$$
\pi_{1}^{\sigma}(u)=\|\tilde{u}\|
$$

Proof. Let us see first that $(1, \sigma)$-absolutely continuous operators are $R S$ abstract 1-summing for suitable $R$ and $S$. Take the functions $S: \mathcal{L}(X, Y) \times$ $X \times \mathbb{R} \rightarrow \mathbb{R}^{+}$given by $S(u, x, a):=\|u(x)\|^{1 /(1-\sigma)}|a|$, and $R: B_{X^{*}} \times X \times \mathbb{R} \rightarrow \mathbb{R}^{+}$ by $R\left(x^{\prime}, x, b\right):=\left|\left\langle x, x^{\prime}\right\rangle\right|\|x\|^{\sigma /(1-\sigma)}|b|$.

For this $R$ and $S$, an operator $u: X \rightarrow Y$ is $R S$-abstract 1-summing if and only if there is a constant $C>0$ such that for each pair of finite sets $x_{1}, \ldots, x_{n} \in X$ and $b_{1}, \ldots, b_{n} \in \mathbb{R}$,

$$
\begin{aligned}
\sum_{i=1}^{n}\left\|u\left(x_{i}\right)\right\|^{1 /(1-\sigma)}\left|b_{i}\right| & =\sum_{i=1}^{n} S\left(u, x_{i}, b_{i}\right) \\
& \leq C \sup _{x^{\prime} \in B_{X^{*}}} \sum_{i=1}^{n} R\left(x^{\prime}, x_{i}, b_{i}\right) \\
& =C \sup _{x^{\prime} \in B_{X^{*}}} \sum_{i=1}^{n}\left|\left\langle x_{i}, x^{\prime}\right\rangle\right|\left\|x_{i}\right\|^{\sigma /(1-\sigma)}\left|b_{i}\right|,
\end{aligned}
$$


that is, if the operator is $(1, \sigma)$-absolutely continuous.

The second step of the proof consists of defining maps $\tilde{R}$ and $\tilde{S}$ in order to apply Theorem 3 . Let $\mathcal{M}(\mu, X)$ be the set of all strongly measurable functions with values in $X$. Define now $\tilde{S}: \mathcal{L}(X, Y) \times \mathcal{M}(\mu, X) \times\left\{\chi_{\Omega}\right\} \times \Omega \rightarrow \mathbb{R}^{+}$given by

$$
\tilde{S}\left(u, f, \chi_{\Omega}, \omega\right):=S\left(u, f(\omega), \chi_{\Omega}(\omega)\right)=\|u \circ f(\omega)\|^{1 /(1-\sigma)},
$$

and $\tilde{R}: B_{X^{*}} \times \mathcal{M}(\mu, X) \times\left\{\chi_{\Omega}\right\} \times \Omega \rightarrow \mathbb{R}^{+}$by

$$
\tilde{R}\left(x^{\prime}, f, \chi_{\Omega}, \omega\right):=R\left(x^{\prime}, f(\omega), \chi_{\Omega}(\omega)\right)=\left|\left\langle f(\omega), x^{\prime}\right\rangle\right|\|f\|^{\sigma /(1-\sigma)} .
$$

An application of Theorem 3 shows that $u$ is $(1, \sigma)$-absolutely continuous if, and only if,

$$
\int_{\Omega}\|u \circ f(w)\|^{1 /(1-\sigma)} d \mu(w) \leq C \sup _{x^{\prime} \in B_{X^{*}}} \int_{\Omega}\left|\left\langle f(w), x^{\prime}\right\rangle\right|\|f(w)\|^{\sigma /(1-\sigma)} d \mu(w),
$$

for all simple function $f \in \mathcal{M}(\mu, X)$. Note that for this case, the proof of Theorem 3 provides that $C^{1-\sigma}=\pi_{1}^{\sigma}(u)$.

Thus, $u$ is $(1, \sigma)$-absolutely continuous if, and only if, $u_{0}: \mathcal{S}_{1 /(1-\sigma)}^{\sigma}(\mu, X) \rightarrow$ $\mathcal{B}_{1 /(1-\sigma)}(\mu, Y)$, given by $u_{0}(f):=u \circ f$, is continuous. Indeed, from (3) we have

$$
\begin{aligned}
& \left(\int_{\Omega}\|u \circ f(w)\|^{1 /(1-\sigma)} d \mu(w)\right)^{1-\sigma} \\
& \leq C^{1-\sigma}\left(\sup _{x^{\prime} \in B_{X^{*}}} \int_{\Omega}\left|\left\langle f(w), x^{\prime}\right\rangle\right|\|f(w)\|^{\sigma /(1-\sigma)} d \mu(w)\right)^{1-\sigma}
\end{aligned}
$$

and since the expression in the left is a norm, we also have for $f=\sum_{i=1}^{n} f_{i}$,

$$
\begin{aligned}
& \left(\int_{\Omega}\left\|u \circ \sum_{i=1}^{n} f_{i}(w)\right\|^{1 /(1-\sigma)} d \mu(w)\right)^{1-\sigma} \\
& \leq \sum_{i=1}^{n}\left(\int_{\Omega}\left\|u \circ f_{i}(w)\right\|^{1 /(1-\sigma)} d \mu(w)\right)^{1-\sigma} \\
& \leq \sum_{i=1}^{n} C^{1-\sigma}\left(\sup _{x^{\prime} \in B_{X^{*}}} \int_{\Omega}\left|\left\langle f_{i}(w), x^{\prime}\right\rangle\right|\left\|f_{i}(w)\right\|^{\sigma /(1-\sigma)} d \mu(w)\right)^{1-\sigma},
\end{aligned}
$$

and thus

$$
\begin{aligned}
& \left(\int_{\Omega}\|u \circ f(w)\|^{1 /(1-\sigma)} d \mu(w)\right)^{1-\sigma} \\
& \leq C^{1-\sigma} \inf _{f=\sum_{i=1}^{n} f_{i}} \sum_{i=1}^{n}\left(\sup _{x^{\prime} \in B_{X^{*}}} \int_{\Omega}\left|\left\langle f_{i}(w), x^{\prime}\right\rangle\right|\left\|f_{i}(w)\right\|^{\sigma /(1-\sigma)} d \mu(w)\right)^{1-\sigma} \\
& =C^{1-\sigma}\|f\|_{1 / 1-\sigma, \sigma} .
\end{aligned}
$$


A standard argument using these computations and the definitions of the constants involved gives also $\left\|u_{0}\right\|=C^{1-\sigma}=\pi_{1}^{\sigma}(u)$.

Note that $u_{0}(f)=\tilde{u}(f)$ for all $f$ in $\mathcal{S}_{1 /(1-\sigma)}^{\sigma}(\mu, X)$. The last step of the proof consists of proving that the composition operator $u_{0}: \mathcal{S}_{1 /(1-\sigma)}^{\sigma}(\mu, X) \rightarrow$ $\mathcal{B}_{1 /(1-\sigma)}(\mu, Y)$ can be extended to the whole space $\mathcal{P}_{1 /(1-\sigma)}^{\sigma}(\mu, X)$, i.e. that the operator $\tilde{u}: \mathcal{P}_{1 /(1-\sigma)}^{\sigma}(\mu, X) \rightarrow \mathcal{B}_{1 /(1-\sigma)}(\mu, Y)$ is well-defined and continuous. To see this, note that the operator $u_{0}: \mathcal{S}_{1 /(1-\sigma)}^{\sigma}(\mu, X) \rightarrow \mathcal{B}_{1 /(1-\sigma)}(\mu, Y)$ can be extended by continuity to an operator $U: \mathcal{P}_{1 /(1-\sigma)}^{\sigma}(\mu, X) \rightarrow \mathcal{B}_{1 /(1-\sigma)}(\mu, Y)$, since $\mathcal{P}_{1 /(1-\sigma)}^{\sigma}(\mu, X)$ is a subspace of the completion of $\mathcal{S}_{1 /(1-\sigma)}^{\sigma}(\mu, X)$. We have to show that $U(f)=\tilde{u}(f)$ for all $f$ in $\mathcal{P}_{1 /(1-\sigma)}^{\sigma}(\mu, X)$. Fix $f \in \mathcal{P}_{1 /(1-\sigma)}^{\sigma}(\mu, X)$ and take a sequence $\left(f_{n}\right) \in \mathcal{S}_{1 /(1-\sigma)}^{\sigma}(\mu, X)$ such that $f_{n} \rightarrow f$ in the norm $\|\cdot\|_{\mathcal{P}_{1 /(1-\sigma)}^{\sigma}}$ of $\mathcal{P}_{1 /(1-\sigma)}^{\sigma}(\mu, X)$. Since $\mathcal{P}_{1 /(1-\sigma)}^{\sigma}(\mu, X) \subseteq \mathcal{P}_{1}(\mu, X)$ continuously, the sequence $\left(f_{n}\right)$ converges to $f$ for the norm $\|\cdot\|_{\mathcal{P}_{1}}$. As $\tilde{u}: \mathcal{P}_{1}(\mu, X) \rightarrow \mathcal{P}_{1}(\mu, Y)$ is continuous, $\left(\tilde{u}\left(f_{n}\right)\right)$ converges to the function $\tilde{u}(f) \in \mathcal{P}_{1}(\mu, Y)$. It follows that

$U(f)=\|\cdot\|_{\mathcal{P}_{1 /(1-\sigma)}^{\sigma}}-\lim _{n} U\left(f_{n}\right)=\|\cdot\|_{\mathcal{P}_{1}}-\lim _{n} U\left(f_{n}\right)=\|\cdot\|_{\mathcal{P}_{1}}-\lim _{n} \tilde{u}\left(f_{n}\right)=\tilde{u}(f)$,

and so, $\tilde{u}(f)$ is Bochner $1 /(1-\sigma)$-integrable. Note also that $\pi_{1}^{\sigma}(u)=\left\|u_{0}\right\|=\|\tilde{u}\|$. This gives the result.

Let us finish this section with two relevant examples.

Example 6 Let $K$ be a compact Hausdorff topological space, and consider the space $C(K)$ of all continuous functions defined on it endowed with the usual sup norm. If $1 \leq p \leq \infty$ and $\nu$ is a measure, we denote as usual by $L_{p}(\nu)$ the space of all (classes of equivalence of) p-integrable functions.

(1) Assume that $\nu$ is a regular Borel probability measure on $K$ and consider the canonical p-summing identification map $j_{p}: C(K) \rightarrow L_{p}(\nu)$. By Lemma 2 the mapping $j_{p}$ is $(1, \sigma)$-absolutely continuous for any $0 \leq \sigma \leq 1$. Let us show how $j_{p}$ improves integrability by a straightforward application of Theorem 5. Let $\sigma=1 / p^{\prime}$ (that is, $p=1 /(1-\sigma)$ ) and consider a strongly measurable function $f: \Omega \rightarrow C(K)$. If $f$ belongs to $\mathcal{P}_{p}^{\sigma}(\mu, C(K))$ then, $j_{p} \circ f: \Omega \rightarrow L_{p}(\nu)$ belongs to $\mathcal{B}_{p}\left(\mu, L_{p}(\nu)\right)$.

(2) Take $\nu$ any $\sigma$-finite measure. Let $1 \leq r \leq 2$ and $2 \leq q<\infty$, and define $\sigma_{0}:=1 / q^{\prime}$. Then $\tilde{u}: \mathcal{P}_{q}^{\sigma_{0}}(\mu, C(K)) \rightarrow \mathcal{B}_{q}\left(\mu, L_{r}(\nu)\right)$ is well-defined and continuous for any $u \in \mathcal{L}\left(C(K), L_{r}(\nu)\right)$. In fact, from [4, Th.3.5] we know that $\mathcal{L}\left(C(K), L_{r}(\nu)\right)=\Pi_{2}\left(C(K), L_{r}(\nu)\right)$. Since $1 /\left(1-\sigma_{0}\right)=q \geq 2$, by Lemma 2 we obtain

$$
\Pi_{2}\left(C(K), L_{r}(\nu)\right) \subseteq \Pi_{q}\left(C(K), L_{r}(\nu)\right) \subseteq \Pi_{1}^{\sigma_{0}}\left(C(K), L_{r}(\nu)\right)
$$

and then Theorem 5 gives the result. 


\section{Applications: $(p, q)$-summing operators and in- tegrability}

The following inclusion property will be useful in this section. It follows easily from the definitions; note that the case $\sigma_{2}=1$ gives the Bochner $p$-norm. Recall that we are considering a finite and non-atomic measure space $(\Omega, \Sigma, \mu)$.

Lemma 7 Let $1 \leq p<\infty$ and $0 \leq \sigma_{1} \leq \sigma_{2} \leq 1$. For a simple function $f$, $\Phi_{p, \sigma_{1}}(f) \leq \Phi_{p, \sigma_{2}}(f)$. Consequently, $\mathcal{P}_{p}^{\sigma_{2}}(\mu, X) \subseteq \mathcal{P}_{p}^{\sigma_{1}}(\mu, X)$ with continuous inclusion.

Proof. Since for every $x^{\prime} \in B_{X^{*}}$, we have that $\left|\left\langle f(w), x^{\prime}\right\rangle\right| \leq\|f(w)\|$, the inequality

$$
\begin{aligned}
& \Phi_{p, \sigma_{1}}(f)^{p}=\sup _{x^{\prime} \in B_{X^{*}}} \int\left(\left|\left\langle f(w), x^{\prime}\right\rangle\right|^{1-\sigma_{1}}\|f(w)\|^{\sigma_{1}}\right)^{p} d \mu \\
= & \sup _{x^{\prime} \in B_{X^{*}}} \int\left(\left|\left\langle f(w), x^{\prime}\right\rangle\right|^{1-\sigma_{2}}\left|\left\langle f(w), x^{\prime}\right\rangle\right|^{\sigma_{2}-\sigma_{1}}\|f(w)\|^{\sigma_{1}}\right)^{p} d \mu \\
\leq & \sup _{x^{\prime} \in B_{X^{*}}} \int\left(\left|\left\langle f(w), x^{\prime}\right\rangle\right|^{1-\sigma_{2}}\|f(w)\|^{\sigma_{2}-\sigma_{1}}\|f(w)\|^{\sigma_{1}}\right)^{p} d \mu \\
= & \sup _{x^{\prime} \in B_{X^{*}}} \int\left(\left|\left\langle f(w), x^{\prime}\right\rangle\right|^{1-\sigma_{2}}\|f(w)\|^{\sigma_{2}}\right)^{p} d \mu=\Phi_{p, \sigma_{2}}(f)^{p}
\end{aligned}
$$

holds. The continuous inclusion in the statement is a direct consequence of this inequality and the definitions.

The following three results are direct applications of Theorem 5 under the hypothesis of coincidences among the operator ideals $\Pi_{p}, \Pi_{1}^{\sigma}$ and $\Pi_{\frac{1}{1-\sigma}, 1}$. They are the source of a lot of particular applications, that will be written in the next subsection.

Corollary 8 Let $1 \leq s<\infty$ and $\sigma=1 / s^{\prime}$. If $u \in \Pi_{s}(X, Y)$ then $\tilde{u}$ : $\mathcal{P}_{s}^{\sigma}(\mu, X) \rightarrow \mathcal{B}_{s}(\mu, Y)$ is well-defined and continuous. In general, if $u \in \Pi_{s}(X, Y)$ and $1 / s^{\prime} \leq \sigma_{1}$, then $\tilde{u}: \mathcal{P}_{s}^{\sigma_{1}}(\mu, X) \rightarrow \mathcal{B}_{s}(\mu, Y)$ is well-defined and continuous.

Proof. The case $s=1$ reduces to Diestel's theorem. Now, note that $s=$ $1 /(1-\sigma)$. Then by Lemma 2 , if $u \in \Pi_{s}(X, Y)$, we have that $u \in \Pi_{1}^{\sigma}(X, Y)$. By Theorem 5 we have then that the operator

$$
\tilde{u}: \mathcal{P}_{s}^{\sigma}(\mu, X)=\mathcal{P}_{1 /(1-\sigma)}^{\sigma}(\mu, X) \rightarrow \mathcal{B}_{1 /(1-\sigma)}(\mu, Y)=\mathcal{B}_{s}(\mu, Y)
$$

is well-defined and continuous. The second statement is a consequence of Lemma 7 , since $\mathcal{P}_{s}^{\sigma_{1}}(\mu, X) \subseteq \mathcal{P}_{s}^{\sigma}(\mu, X)$ continuously.

The next results are obvious, we write them as corollaries for further use.

Corollary 9 Let $1 \leq p<\infty$ and $\sigma=1 / p^{\prime}$. Assume that $\Pi_{p, 1}(X, Y)=$ $\Pi_{1}^{\sigma}(X, Y)$. Then $u \in \Pi_{p, 1}(X, Y)$ if and only if $\tilde{u}: \mathcal{P}_{p}^{\sigma}(\mu, X) \rightarrow \mathcal{B}_{p}(\mu, Y)$ is well-defined and continuous. 
Corollary 10 Let $1 \leq p \leq q<\infty$ and $\sigma=1 / q^{\prime}$. Assume that $\mathcal{L}(X, Y)=$ $\Pi_{p}(X, Y)$. Then $\tilde{u}: \mathcal{P}_{q}^{\sigma}(\mu, X) \rightarrow \mathcal{B}_{q}(\mu, Y)$ is well-defined and continuous for any $u \in \mathcal{L}(X, Y)$.

Proof. It follows from the inclusions $\Pi_{p}(X, Y) \subset \Pi_{q}(X, Y) \subset \Pi_{1, \sigma}(X, Y)$ (Lemma 2) and Theorem 5.

In what follows we deal with classical Banach spaces. We will use some results on coincidence of some classical operator ideals with the interpolated class of the ideals of $(p, \sigma)$-absolutely continuous operators; our main source is the paper [10]. We will also use some classical results.

\subsection{Operators improving integrability of Hilbert space val- ued functions}

Consider a Hilbert space $L^{2}$. It is well-known (see [4, Corollary 11.16]) that for each $1 \leq p<\infty, \Pi_{p}\left(L^{2}, L^{2}\right)=\Pi_{1}\left(L^{2}, L^{2}\right)$. Let $1 \leq p<\infty$ and let $\mathcal{A}_{p}\left(L^{2}, L^{2}\right)$ be the corresponding component of the ideal of the $p$-approximable operators. It is well-known that $\Pi_{2}\left(L^{2}, L^{2}\right)=\mathcal{A}_{2}\left(L^{2}, L^{2}\right)$ (see e.g. [6, Theorem 20.5.1] or $[4$, Theorem 4.10]). In this setting, we can obtain the following

Corollary 11 Let $\mu$ be a non-atomic finite positive measure. Let $1 \leq p<\infty$ and $\sigma=1 / p^{\prime}$ and consider $u: L^{2} \rightarrow L^{2}$ a $2 p$-approximable operator. Then the composition operator $\tilde{u}: \mathcal{P}_{p}^{\sigma}\left(\mu, L^{2}\right) \rightarrow \mathcal{B}_{p}\left(\mu, L^{2}\right)$ is well defined and continuous.

Proof. As a consequence of [10, Prop.5.1] and the comments above, we know that for every $0 \leq \sigma<1, \Pi_{1}^{\sigma}\left(L^{2}, L^{2}\right)=\Pi_{2}^{\sigma}\left(L^{2}, L^{2}\right)=\mathcal{A}_{\frac{2}{1-\sigma}}\left(L^{2}, L^{2}\right)$. Theorem 5 gives the result.

\subsection{Operators improving integrability of $\mathcal{L}^{\infty}$ and $C(K)$ val- ued functions}

Consider a space $L^{\infty}(\nu)$ for any measure $\nu$. By [10, Prop.5.2], for every Banach space $E$ and each $1 \leq p<\infty, 0 \leq \sigma<1$ and $\varepsilon>0$, we have that

$$
\Pi_{\frac{p}{1-\sigma}}\left(L^{\infty}, E\right) \subseteq \Pi_{p}^{\sigma}\left(L^{\infty}, E\right) \subseteq \Pi_{\frac{p}{1-\sigma}+\varepsilon}\left(L^{\infty}, E\right) .
$$

This provides (see (1) below) a partial converse to Corollary 8, which we reproduce for $L^{\infty}$-spaces in part (2) of the next result.

Corollary 12 Fix $1 \leq q<p<\infty, \sigma=1 / q^{\prime}$, and consider a linear operator $u: L^{\infty}(\mu) \rightarrow E$.

(1) If $u$ satisfies that its associated composition operator $\tilde{u}$ is well-defined and continuous from $\mathcal{P}_{q}^{\sigma}\left(\mu, L^{\infty}\right)$ to $\mathcal{B}_{q}(\mu, E)$, then $u$ is p-summing.

(2) In the case that $u$ is q-summing, then $\tilde{u}$ is well-defined and continuous from $\mathcal{P}_{q}^{\sigma}\left(\mu, L^{\infty}\right)$ to $\mathcal{B}_{q}(\mu, E)$. 
Proof. For the proof of (1), just note that by Theorem 5, $u$ belongs to $\Pi_{1}^{\sigma}\left(L^{\infty}, E\right)$. The right hand side inclusion before the corollary gives (1). Item (2) is a particular case of Corollary 8.

As a consequence of a result of Pisier, it can be proved that the classes of $\left(\frac{1}{1-\sigma}, 1\right)$-summing operators and the class of $(1, \sigma)$-absolutely continuous operators coincide on $C(K)$-spaces (see [14, Th.2.4 (ii)] and the characterization of $(p, \sigma)$-absolutely continuous operators given by [10, Th.4.1 (ii)] for the particular case of $C(K)$-spaces; see also [8]). Recall that a consequence of Pisier's result is that for $1 \leq q<p<\infty$, the $(p, q)$-summing operators acting in $C(K)$-spaces coincide with the $(p, 1)$-summing ones. Thus, for a compact Hausdorff space $K$ and a Banach space $E$, we have the following

Corollary 13 Let $1 \leq q<p<\infty$ and let $\sigma=1 / p^{\prime}$. An operator $u: C(K) \rightarrow E$ is $(p, q)$-summing if and only if the composition operator $\tilde{u}$ from $\mathcal{P}_{p}^{\sigma}(\mu, C(K))$ to $\mathcal{B}_{p}(\mu, E)$ is well defined and continuous.

\subsection{Operators improving integrability of $\mathcal{L}^{1}$-spaces valued functions}

The case of $(p, \sigma)$-absolutely continuous operators from $\mathcal{L}^{1}$-spaces has been intensively studied (see $[10, \S 6]$ ), so we can find a lot of applications of our results in this case. Let $0 \leq \sigma<1$. Following the notation in this paper -and taking into account the comments just after the definition in [10, page 201]-, we define

$$
H_{\sigma}=:\left\{E \text { Banach }: \mathcal{L}\left(L^{1}, E\right)=\Pi_{1}^{\sigma}\left(L^{1}, E\right) \text { for all } L^{1} \text {-spaces }\right\} .
$$

Corollary 14 Fix an $\mathcal{L}^{1}$-space $L^{1}$ and a non-atomic finite positive measure $\mu$. Let $1 \leq p<\infty$ and $\sigma=1 / p^{\prime}$. Consider a Banach space $E \in H_{\sigma}$. For every operator $u: L^{1} \rightarrow E$, the composition operator $\tilde{u}$ from $\mathcal{P}_{p}^{\sigma}\left(\mu, L^{1}\right)$ to $\mathcal{B}_{p}(\mu, E)$ is well defined and continuous.

Let us apply this general result to some relevant Banach spaces belonging to the class $H_{\sigma}$. First, note that since the ideal $\Pi_{1}^{\sigma}$ is injective ([10, Th.3.2.]) and $\ell^{1}$ has the lifting property all the subspaces of the spaces in $H_{\sigma}$ are again in $H_{\sigma}$. Following the notation of Matter in [10], we say that a Banach space $E$ is $(\sigma, p)$-Hilbertian $\left(\sigma\right.$-Hilbertian) if there is an interpolation pair $\left(H, E_{1}\right)$ with $H$ a Hilbert space and $E_{1}$ a Banach space such that $E=\left(H, E_{1}\right)_{\sigma, p}$ $\left(E=\left[H, E_{1}\right]_{\sigma}\right)$ isomorphically. Here, $(\cdot, \cdot)_{\sigma, p}$ and $[\cdot, \cdot]_{\sigma}$ denote the usual real and complex interpolation spaces.

Corollary 15 Let $1 \leq p<\infty$ and $\sigma=1 / p^{\prime}$. Fix an $\mathcal{L}^{1}$-space $L^{1}$ and a non-atomic finite positive measure $\mu$. Each of the following Banach spaces $E$ satisfies that for every operator $u: L^{1} \rightarrow E$, the associated composition operator $\tilde{u}: \mathcal{P}_{p}^{\sigma}\left(\mu, L^{1}\right) \rightarrow \mathcal{B}_{p}(\mu, E)$ is well defined and continuous.

(1) E being a quotient of an $L^{\infty}$-space having cotype smaller that $\frac{2}{1-\sigma}$.

(2) $E=L^{r}$ for $2 \leq r<\frac{2}{1-\sigma}$. 
(3) E being a $(\sigma, 2)$-Hilbertian space.

(4) E being a $\sigma$-Hilbertian space.

(5) E being a Lorentz space $L_{r, s}$ for $\frac{2}{1+\sigma}<r$ and $s<\frac{2}{1-\sigma}$.

Proof. (1) Apply [10, Prop.6.5]. (2) Apply [10, Cor.6.6(a)]. (3) [10, Th.8.1]. (4) $[10$, Th.8.2]. (5) $[10$, Th.9.2(a)].

Let us finish the paper with a result regarding super-reflexive Banach lattices. Recall that super-reflexive Banach spaces were the original motivation for introducing the ideal of $(p, \sigma)$-absolutely continuous operators. The following result is an application of our Theorem 5 and Corollary 10.4 in [10].

Corollary 16 Fix an $\mathcal{L}^{1}$-space $L^{1}$ and a non-atomic finite positive measure $\mu$. If $F$ is a super-reflexive Banach lattice, then there is $1 \leq p<\infty$ such that for every operator $u: L^{1} \rightarrow E$ the associated composition operator $\tilde{u}: \mathcal{P}_{p}^{\sigma}\left(\mu, L^{1}\right) \rightarrow$ $\mathcal{B}_{p}(\mu, F)$ is well defined and continuous, for $\sigma=1 / p^{\prime}$.

Acknowledgments: D. Pellegrino acknowledges with thanks the support of CNPq Grant 313797/2013-7 (Brazil). P. Rueda acknowledges with thanks the support of the Ministerio de Economía y Competitividad (Spain) MTM201122417. E.A. Sánchez Pérez acknowledges with thanks the support of the Ministerio de Economía y Competitividad (Spain) MTM2012-36740-C02-02.

\section{References}

[1] G. Botelho; D. Pellegrino; P. Rueda, A unified Pietsch domination theorem. J. Math. Anal. Appl. 365 (2010), no. 1, 269-276.

[2] A. Defant; K. Floret, Tensor norms and operator ideals, North-Holland, Amsterdam, 1992.

[3] J. Diestel, An elementary characterization of absolutely summing operators. Math. Ann. 196 (1972), 101-105.

[4] J. Diestel; H. Jarchow; A. Tonge, Absolutely summing operators. Cambridge University Press, Cambridge, 1995.

[5] J. Farmer; W.B. Johnson, Lipschitz p-summing operators, Proc. Amer. Math. Soc. 137 (2009), 2989-2995.

[6] H. Jarchow, Localy convex, spaces, Teubner, Stuttgart, 1981.

[7] J. A. López Molina; E. A. Sánchez Pérez, Ideales de operadores absolutamente continuos, Rev. Real Acad. Ciencias Exactas, Físicas y Naturales, Madrid 87 (1993), 349-378.

[8] J. A. López Molina; E. A. Sánchez Pérez, The associated tensor norm to $(q, p)$-absolutely summing operators on $C(K)$-spaces, Czec. Math. J. 47(4) (1997), 627-631. 
[9] J.A. López Molina; E.A. Sánchez-Pérez, On operator ideals related to $(p, \sigma)$-absolutely continuous operator, Studia Math. 131(8) (2000), 25-40.

[10] U. Matter, Absolute continuous operators and super-reflexivity, Math. Nachr. 130 (1987), 193-216.

[11] D. Pellegrino; J. Santos, A general Pietsch domination theorem. J. Math. Anal. Appl. 375 (2011), no. 1, 371-374.

[12] D. Pellegrino; J. Santos; J.B. Seoane-Sepúlveda, Some techniques on nonlinear analysis and applications. Adv. Math. 229 (2012), 1235-1265.

[13] A. Pietsch, Operator Ideals. Deutsch. Verlag Wiss., Berlin, 1978; NorthHolland, Amsterdam-London-New York-Tokyo, 1980.

[14] G. Pisier, Factorization of operators through $L_{p \infty}$ or $L_{p 1}$ and noncommutative generalizations. Math. Ann. 276 (1986), no. 1, 105-136.

[15] J. Rodríguez, Absolutely summing operators and integration of vectorvalued functions. J. Math. Anal. Appl. 316 (2006), no. 2, 579-600. 